\title{
Multislice simulation of scanning transmission electron microscope images
}

\author{
C. Dwyer*
}

* Centre for Electron Microscopy, Monash University, Clayton, Victoria 3800, Australia

In the pursuit of quantification in the various transmission electron microscope (TEM) imaging and diffraction methods, the role of simulations is intrinsic. For various practical reasons, most notably its elegant simplicity and efficiency in handling non-periodic atomic structures, the fast Fourier transform- (FFT) based multislice method [1] has become widely adopted.

However, the simulation of scanning transmission electron microscope (STEM) images presents somewhat of a challenge, simply due to the time required to generate data for each position of the probe. This is particularly apparent for non-crystalline specimens. While the FFT multislice method can easily handle non-crystalline specimens by means of a supercell, a straightforward adaptation to STEM, whereby a separate multislice calculation is carried out for each pixel [2] (pixel-by-pixel calculation), can lead to impractical computation times ( $\sim$ weeks).

Such impracticalities have motivated work to eliminate them. In the present work, two approaches for reducing the computation of multislice simulations of STEM images are examined:

The first approach is to compute the STEM image based on Green functions. This approach offers advantages in terms of both efficiency and the ability to quickly re-calculate images for a different set of probe aberrations. This approach will be described and tests of its performance will be presented.

The second approach is to recognize that modern desktop graphics processing units (GPUs) can often provide a significant advance in floating-point performance over modern desktop central processing units (CPUs). The adaptation of a multislice code to run on the GPU will be described, and the performance of three GPUs of varying prices will be presented.

As an example of a large calculation made practical by the Green function approach on the GPU, Fig. 1 shows an ADF-STEM image of a $\beta$-Sn precipitate in an $\alpha$-Al matrix. The field of view is $80 \AA$ x $80 \AA$ and the calculation size is $1024 \times 1024$ pixels. The computation time was $1 \mathrm{hr}$. By comparison, a pixel-by-pixel calculation would have taken 3 days on the same GPU, and 22 days on a modern desktop quad-core CPU running in parallel [3].

References

[1] E. J. Kirkland, Advanced Computing in Electron Microscopy, Plenum Press (1998).

[2] J. Pizzaro et al., Appl. Phys. Lett. 93 (2008) 153107.

[3] The author would like to thank S. D. Findlay and D. Lynch for enthusiastic discussions. 


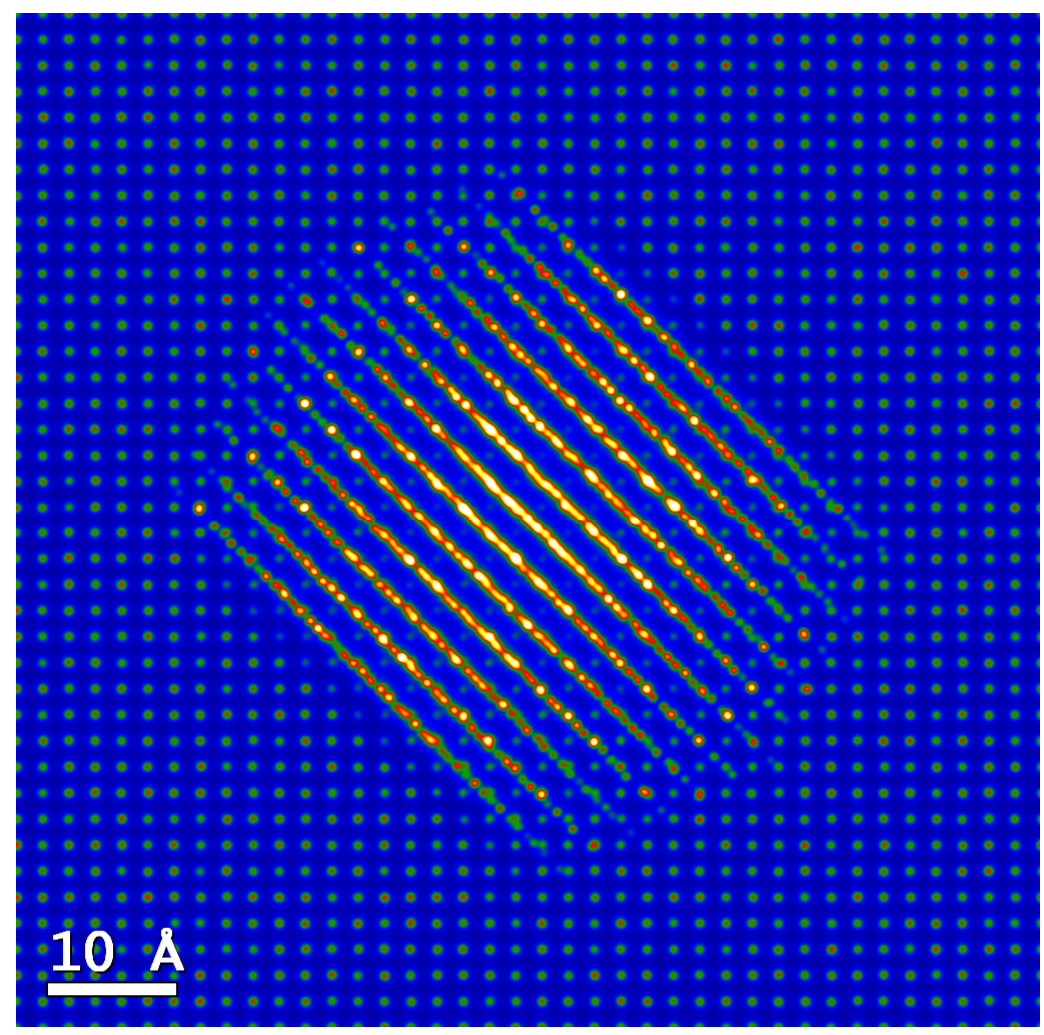

FIG. 1. ADF-STEM image of a $\beta$-Sn precipitate in an $\alpha-\mathrm{Al}$ matrix. The beam energy is $300 \mathrm{keV}$. The probe size is $0.8 \AA$. No lens aberrations were included. 